\title{
Laser action in nanowires: Observation of the transition from amplified spontaneous emission to laser oscillation
}

\section{Citation}

Zimmler, Mariano A., Jiming Bao, Federico Capasso, Sven Müller, and Carsten Ronning. 2008.

"Laser Action in Nanowires: Observation of the Transition from Amplified Spontaneous Emission to Laser Oscillation." Applied Physics Letters 93 (5): 51101. https://doi.org/10.1063/1.2965797.

\section{Permanent link}

http://nrs.harvard.edu/urn-3:HUL.InstRepos:41371598

\section{Terms of Use}

This article was downloaded from Harvard University's DASH repository, and is made available under the terms and conditions applicable to Other Posted Material, as set forth at http:// nrs.harvard.edu/urn-3:HUL.InstRepos:dash.current.terms-of-use\#LAA

\section{Share Your Story}

The Harvard community has made this article openly available.

Please share how this access benefits you. Submit a story.

Accessibility 


\title{
Laser action in nanowires: Observation of the transition from amplified spontaneous emission to laser oscillation
}

\author{
Mariano A. Zimmler, ${ }^{1}$ Jiming Bao, ${ }^{1}$ Federico Capasso, ${ }^{1, a)}$ Sven Müller, ${ }^{2}$ and \\ Carsten Ronning ${ }^{3}$ \\ ${ }^{1}$ School of Engineering and Applied Sciences, Harvard University, Cambridge, Massachusetts 02138, USA \\ ${ }^{2}$ Institute of Physics, University of Göttingen, 37077 Göttingen, Germany \\ ${ }^{3}$ Institute for Solid State Physics, University of Jena, 00743 Jena, Germany
}

(Received 2 April 2008; accepted 21 May 2008; published online 4 August 2008)

\begin{abstract}
Direct evidence of the transition from amplified spontaneous emission to laser action in optically pumped zinc oxide $(\mathrm{ZnO})$ nanowires, at room temperature, is presented. The optical power evolves from a superlinear to a linear regime as the pump power exceeds threshold, concomitant with a transition to directional emission along the nanowire and the emergence of well defined cavity Fabry-Pérot modes around a wavelength of $\approx 385 \mathrm{~nm}$, the intensity of which exceeds the spontaneous emission background by orders of magnitude. The laser oscillation threshold is found to be strongly dependent on nanowire diameter, with no laser oscillation observed for diameters smaller than $\sim 150 \mathrm{~nm}$. Finally, we use an alternative "head on" detection geometry to measure the output power of a single nanowire laser. (C) 2008 American Institute of Physics.
\end{abstract}

[DOI: $10.1063 / 1.2965797]$

Semiconductor nanowires have recently been proposed as the next frontier in the miniaturization of semiconductor lasers. While previous reports provided a clear evidence of amplified spontaneous emission (ASE) in zinc oxide $(\mathrm{ZnO}){ }^{1-5}$ gallium nitride $(\mathrm{GaN}){ }^{6}$ and cadmium sulfide $(\mathrm{CdS})^{7,8}$ semiconductor nanowires and some evidence of lasing in gallium antimonide $(\mathrm{GaSb}){ }^{9}$ the conclusive demonstration of laser oscillation has remained elusive. In particular, no laser threshold marking the transition between a superlinear region, characteristic of ASE, and a linear region, characteristic of laser oscillation, ${ }^{10}$ with increasing pump power or drive current, has yet been reported. In addition, the highly directional emission characteristic of lasers could not be observed since only light scattered from the nanowire ends was measured.

Laser oscillation occurs when the round-trip gain matches the round-trip losses; ${ }^{10}$ in nanowires the latter are typically dominated by the mirror losses (i.e., transmission at the end facets). The reason for this is that in nanowires with diameters comparable to or smaller than the wavelength of light in the semiconductor diffraction causes the optical field to "spill out" of the nanowire. This contributes to the losses since only the fraction of the optical field inside the nanowire experiences a refractive index contrast at the end facets, thus reducing the amount of reflection. Here we present unambiguous evidence of optically pumped laser oscillation at ultraviolet frequencies in individual $\mathrm{ZnO}$ nanowires at room temperature. By means of systematic measurements on nanowires of different dimensions, we demonstrate the existence of a critical diameter below which no lasing occurs. Also, using an alternative "head on" detection geometry, we measure the output power of a single nanowire laser.

Single-crystal $\mathrm{ZnO}$ nanowires were synthesized by a simple vapor transport technique ${ }^{11}$ and subsequently dispersed onto a $\mathrm{Si} / \mathrm{SiO}_{2}$ substrate (500 $\mathrm{nm}$ thermal oxide). Individual nanowires were optically excited with the

${ }^{\text {a)} E l e c t r o n i c ~ m a i l: ~ c a p a s s o @ ~ @ e a s . h a r v a r d . e d u . ~}$ frequency-tripled output $(355 \mathrm{~nm})$ of a Nd:YAG (yttrium aluminum garnet) laser ( $500 \mathrm{~Hz}, 6 \mathrm{~ns}$ pulse length) focused to a diameter of $\sim 100 \mu \mathrm{m}$. The emission from individual nanowires was collected by a reflective microscope objective and coupled to a $55 \mathrm{~cm}$ spectrometer and a charge coupled device $(\mathrm{CCD})$ camera. All measurements were performed at room temperature.

Figures 1(a) and 1(b) show the optical spectra and the corresponding $\mathrm{CCD}$ images, respectively, for a $\mathrm{ZnO}$ nanowire as a function of pump intensity $I_{\mathrm{ex}}$. For $I_{\mathrm{ex}}$ $\$ 200 \mathrm{~kW} \mathrm{~cm}{ }^{-2}$, the spectra are broad and featureless, centered around $\sim 382 \mathrm{~nm}$ and with a full width at half maximum (FWHM) of $19.3 \mathrm{~nm}$. In this regime, light is emitted essentially isotropically along the nanowire [Fig. 1(b)], and the output power (Fig. 2) depends linearly on the excitation intensity, consistent with spontaneous emission. For

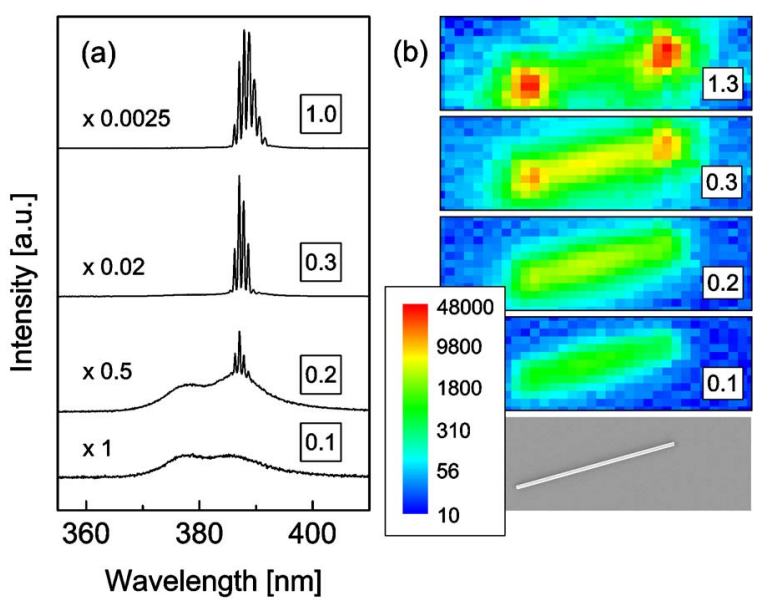

FIG. 1. (Color online) Laser oscillation in $\mathrm{ZnO}$ nanowires. (a) Output spectra vs pump intensity of a $12.2 \mu \mathrm{m}$ long $250 \mathrm{~nm}$ diameter $\mathrm{ZnO}$ nanowire. (b) Scanning electron microscopy and CCD images under different pump intensities for the same nanowire as in (a). The labels indicate the pump intensity in units of $\mathrm{MW} \mathrm{cm}^{-2}$. The color scale indicates the number of counts. 


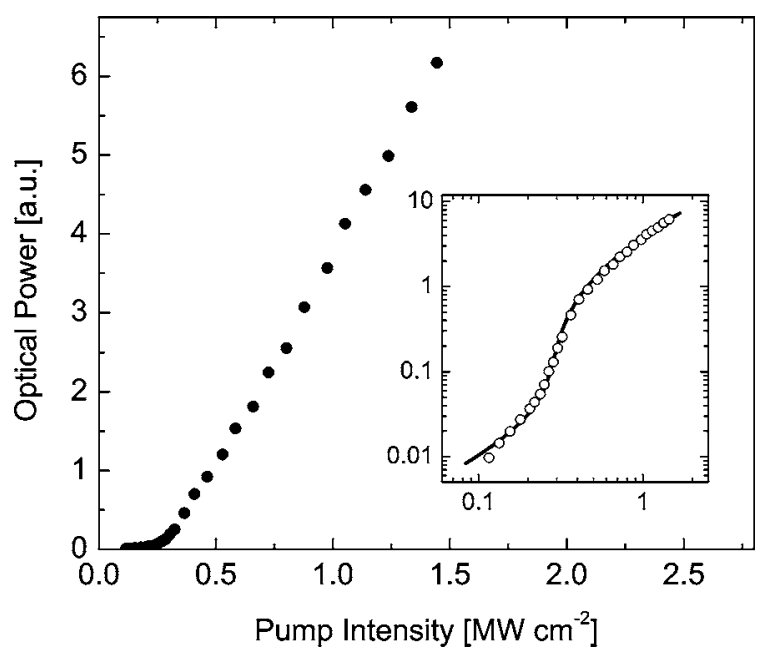

FIG. 2. Laser oscillation in $\mathrm{ZnO}$ nanowires. Pump intensity dependence of the total output power (circles) for the same nanowire as in Fig. 1. The optical power was collected from the scattered light at one of the nanowire ends. The inset shows the same data on a log-log scale as well as a fit to the data using the model in Ref. 12.

$200 \mathrm{~kW} \mathrm{~cm}^{-2} \leq I_{\mathrm{ex}} \lessgtr 300 \mathrm{~kW} \mathrm{~cm}^{-2}$, the spectra consist of a broad emission with the addition of sharp (FWHM $<0.4 \mathrm{~nm}$ ) emission lines. In this regime, population inversion starts building up, leading to ASE along the nanowire at wavelengths corresponding to the longitudinal modes and thus to the enhanced emission from the nanowire ends [Fig. 1(b)]. Furthermore, the output power exhibits a superlinear increase with pump intensity [Fig. 2], which is the expected behavior as the laser threshold is approached. ${ }^{10,12}$ For $I_{\mathrm{ex}}$ $\gtrsim 300 \mathrm{~kW} \mathrm{~cm}{ }^{-2}$, the spectra are dominated by sharp emission lines; their intensity is orders of magnitude greater than the spontaneous emission background. The output power depends linearly on excitation intensity [Fig. 2] and is concentrated in a narrow emission range $(385 \mathrm{~nm}<\lambda<390 \mathrm{~nm})$. The inset in Fig. 2 shows the output power on a log-log scale as well as a fit (solid line) with a multimode laser model, ${ }^{12}$ which gives a threshold of $\simeq 270 \mathrm{~kW} \mathrm{~cm}{ }^{-2}$. ${ }^{12}$ Note that the threshold in semiconductor lasers is "softer" than that in other lasers due to the small cavity volume and to the relatively high levels of spontaneous emission. ${ }^{10,12}$ In summary, the data in Figs. 1 and 2 show the expected behavior of the output power of the nanowire device as it passes through the threshold for laser oscillation.

The sharp features observed in Fig. 1(a) correspond to longitudinal Fabry-Pérot modes. The mode spacing in a Fabry-Pérot cavity is given by ${ }^{10} \Delta \lambda=(1 / L)\left[\left(\lambda^{2} / 2\right)(n\right.$ $-\lambda d n / d \lambda)^{-1}$ ], where $L$ is the cavity length and $n$ is the index of refraction at wavelength $\lambda$. Thus, for a fixed $\lambda$, the mode spacing $\Delta \lambda$ should scale in proportion to the inverse length $1 / L$. This behavior is illustrated in Fig. 3, which shows above-threshold spectra for nanowires of different lengths and a plot of $\Delta \lambda$ for nine nanowires. The slope of the fit is $10765( \pm 1.7 \%) \mathrm{nm}^{2}$. With $\lambda=385 \mathrm{~nm}$ and $n=2.4$, this gives $d n / d \lambda \approx-0.012 \mathrm{~nm}^{-1}$. This value is in reasonable agreement with published data for $\mathrm{ZnO},{ }^{13}$ which gives $d n / d \lambda \approx$ $-0.015 \mathrm{~nm}^{-1}$.

The threshold condition for laser oscillation is that the round-trip gain inside the nanowire must equal the round-trip losses, ${ }^{10}$
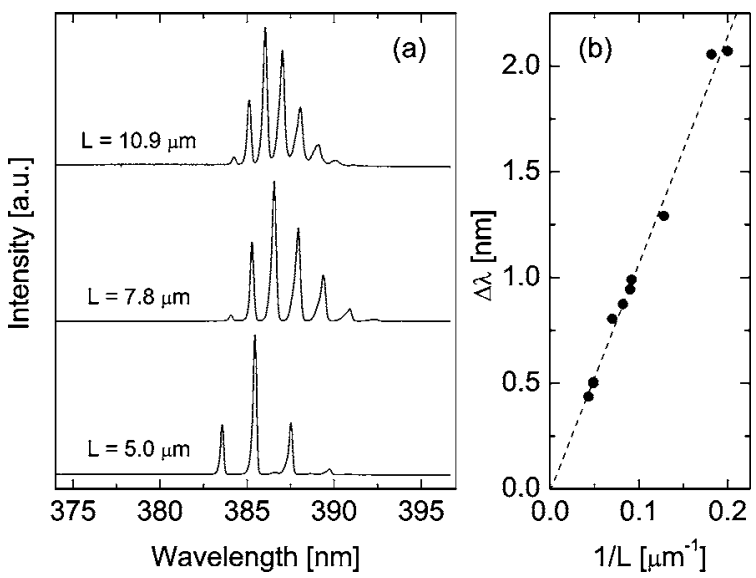

FIG. 3. Nanowire length dependence of above-threshold laser spectra for $\mathrm{ZnO}$ nanowires. (a) Laser oscillation spectra for three nanowires of different lengths. (b) Spacing between adjacent modes vs $1 / L$. Note that the linear fit extrapolates to $0 \mathrm{~nm}$ spacing for $L \rightarrow \infty$.

$$
\Gamma g_{\text {th }}=\alpha_{w}+\alpha_{m}, \quad \alpha_{m}=\frac{1}{L} \ln \frac{1}{R},
$$

where $\Gamma$ is the confinement factor, i.e., the fraction of the mode intensity contained within the nanowire, $g_{\text {th }}$ is the material gain, $\alpha_{w}$ is the waveguide loss, $\alpha_{m}$ accounts for the losses at the end facets (i.e., the mirror losses), $L$ is the length of the nanowire, and $R$ is the reflection coefficient, assumed the same for both laser ends. In contrast to conventional edge emitting semiconductor lasers where typically $\alpha_{w}>\alpha_{m}$, in nanowire lasers, $\alpha_{m} \gg \alpha_{w}$ due to the much smaller cavity length and the smaller reflection coefficient. ${ }^{14}$ As a result, the threshold gain is a strong function of $L$ and the nanowire diameter $D$. Figure 4 shows the devices that lased and the ones that did not (the former ones indicated by filled circles) in the plane defined by two key dimensions: diameter and wire length. Nanowires with diameters smaller than $\sim 150 \mathrm{~nm}$ did not reach threshold, independent of the nanowire length. Qualitatively, this can be explained by the diameter dependence of the reflection coefficient $R$, which is directly related to the confinement factor $\Gamma,{ }^{14}$ as was explained in the introduction. For example, for the most confined mode in our geometry, the fraction of the mode inten-

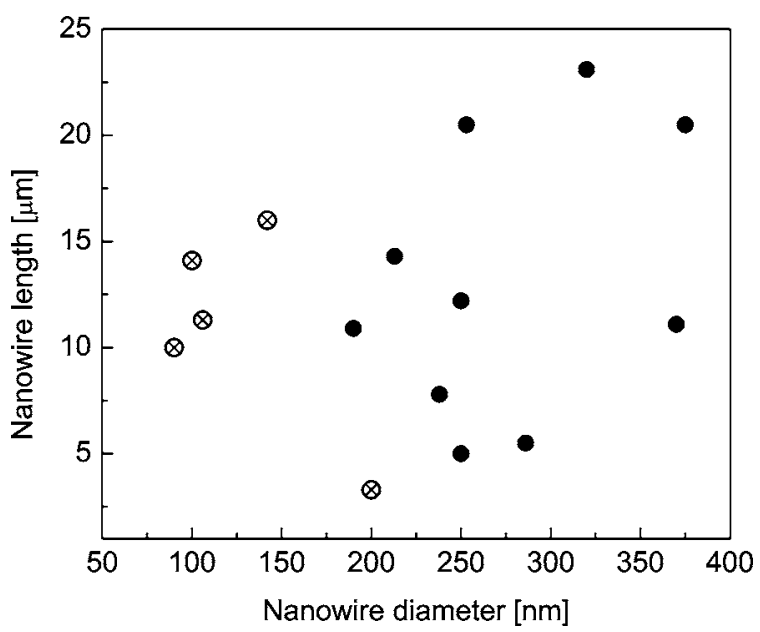

FIG. 4. Experimental results on lasing for nanowires of different dimensions; crosses $(\otimes)$ indicate nanowires which did not lase and circles $(\mathbf{O})$ indicate those which did. 


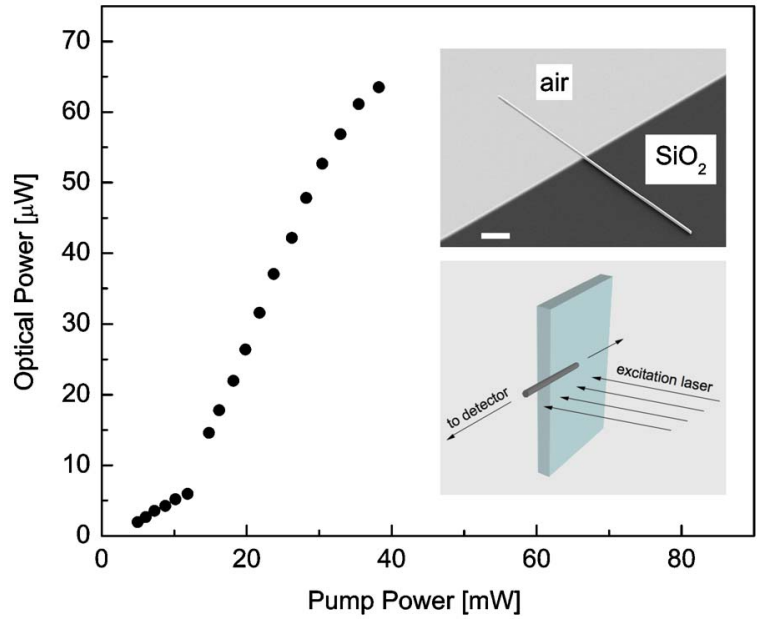

FIG. 5. (Color online) Peak optical power uncorrected for the collection efficiency $(10 \%)$ measured head on at the nanowire end.

sity inside the nanowire decreases from $\sim 85 \%$ for $D$ $=150 \mathrm{~nm}$ to $<1 \%$ for $D=100 \mathrm{~nm}$. The exact calculations need to be performed numerically to account for substrate effects and will be presented in a future publication.

The detection geometry used above only permits collection of a small fraction of the total output power since abovethreshold light is emitted primarily in the direction along the nanowire axis. ${ }^{15}$ In order to measure the output power of a nanowire laser, it is therefore necessary to collect the emission head on. Such an alternative geometry is depicted schematically in Fig. 5: A nanowire is shown partially suspended in air and partially resting on a substrate $(500 \mathrm{~nm}$ of thermal oxide on silicon). The nanowire is then excited uniformly along its entire length, and the emission is collected from one end, at an angle of $90^{\circ}$ from the excitation beam, using a silicon detector. The data in Fig. 5 show the detected peak power versus pump peak power.

In conclusion, we have presented unambiguous evidence of laser action in $\mathrm{ZnO}$ nanowires at room temperature through a systematic study of the evolution from ASE to laser oscillation above threshold. We have demonstrated the key dependence of laser threshold on nanowire diameter and measured the output power of a single nanowire laser using a head on geometry, which provides a useful benchmark for the future development of these nanoscale devices.

This work was supported by the National Science Foundation Nanoscale Science and Engineering Center (NSEC) under Contract No. NSF/PHY 06-46094 and by the Deutsche Forschungsgemeinschaft under Grant No. Ro1198/7. The support of the Center for Nanoscale Systems (CNS) at Harvard University is also gratefully acknowledged. Harvard-CNS is a member of the National Nanotechnology Infrastructure Network (NNIN).

${ }^{1}$ M. H. Huang, S. Mao, H. Feick, H. Yan, Y. Wu, H. Kind, E. Weber, R. Russo, and P. Yang, Science 292, 1897 (2001).

${ }^{2}$ J. C. Johnson, H. Yan, R. D. Schaller, L. H. Haber, R. J. Saykally, and P. Yang, J. Phys. Chem. B 105, 11387 (2001).

${ }^{3}$ S. F. Yu, C. Yuen, S. P. Lau, W. I. Park, and G.-C. Yi, Appl. Phys. Lett. 84, 3241 (2004).

${ }^{4}$ H.-C. Hsu, C.-Y. Wu, and W.-F. Hsieh, J. Appl. Phys. 97, 064315 (2005).

${ }^{5}$ X. Han, G. Wang, Q. Wang, L. Cao, R. Liu, B. Zou, and J. G. Hou, Appl. Phys. Lett. 86, 223106 (2005).

${ }^{6}$ J. C. Johnson, H.-J. Choi, K. P. Knutsen, R. D. Schaller, P. Yang, and R. J. Saykally, Nat. Mater. 1, 106 (2002).

${ }^{7}$ X. Duan, Y. Huang, R. Agarwal, and C. M. Lieber, Nature (London) 421, 241 (2003).

${ }^{8}$ R. Agarwal, C. J. Barrelet, and C. M. Lieber, Nano Lett. 5, 917 (2005).

${ }^{9}$ A. H. Chin, S. Vaddiraju, A. V. Maslov, C. Z. Ning, M. K. Sunkara, and M. Meyyappan, Appl. Phys. Lett. 88, 163115 (2006).

${ }^{10}$ A. E. Siegman, Lasers (University Science Books, Sausalito, CA, 1986).

${ }^{11}$ C. Borchers, S. Müller, D. Stichtenoth, D. Schwen, and C. Ronning, J. Phys. Chem. B 110, 1656 (2006).

${ }^{12}$ L. W. Casperson, J. Appl. Phys. 46, 5194 (1975). The theory in this paper gives the output power of the laser as a function of the normalized pump rate and a parameter $x_{0}$, which is proportional to the mode density [Eq (23)]. An excellent fit of this equation to the data is obtained with $x_{0}=0.016$.

${ }^{13}$ Semiconductors: Data Handbook, edited by O. Madelung (Springer, Berlin, 2003).

${ }^{14}$ A. V. Maslov and C. Z. Ning, Appl. Phys. Lett. 83, 1237 (2003).

${ }^{15}$ A. V. Maslov and C. Z. Ning, Opt. Lett. 29, 572 (2004). 